\title{
Relaciones de género en el campo educativo desde la perspectiva de Bourdieu*
}

\author{
Florencia María Elverdin**
}

\section{Resumen}

El presente trabajo expone el estudio de la relación entre el campo educativo y las diversas concepciones de género que en este tipo de espacios se configuran, particularizando la mirada en la escuela rural de la localidad de Berisso.

Para ello, se comienza por realizar una breve descripción de la posición que ocupa la mujer en la educación, para, posteriormente, analizar los elementos prácticos singulares que la (re)producen, partiendo de la premisa de que, tal como refleja la investigación de campo realizada, las acciones educativas se distinguen según el sexo de los aprendices.

Apoyándome en la mirada sustancialista que se construye en la cotidianidad en la que opera el sentido común, lo natural, lo dado de las relaciones entre dos sexos acompañado de prácticas, se muestra cómo el género (relación entre los sexos) (se) constituye (en) las diferencias y cómo es, a fin de cuentas, una construcción cultural (Bourdieu, 1997; Lamas, 1992; Velásquez, 2003).

\footnotetext{
* Artículo recibido 9 de abril de 2012. Aceptado 9 de diciembre de 2012.

* * Licenciada en Trabajo Social. Colaboradora en el proyecto de investigación Paradojas del diseño e implementación de los planes de empleo en el Gran La Plata. Docente de la materia Investigación Social II, Facultad de Trabajo Social, UNLP. Contacto: florelverdin@hotmail.com

Docente de la cátedra de Metodología de la Investigación Científica, Facultad de Ciencias de la Salud, UCALP, Argentina. Investigación realizada en el marco del Programa de Investigación Movimientos Sociales y Condiciones de Vida; Facultad de Trabajo Social, Universidad Nacional de La Plata.
} 


\section{Palabras clave}

Género - educación - campo - habitus.

\section{Abstract}

The present work exposed the study of relationship between eduacational space and the different conceptions gende that in this space are configure, particularly the view at a rural school os the city of Berisso.

For this, are began for do a brief description of the position of women in education, to, later, analyze the elements practical singular that reproduce, starting of the premise that, such as shows as the research of field did, the educational activities are distinguished to sex.

Supporting me in the subsantialist view which constitutes the everyday in which common sense and the natural operate, and what is given in the relationship between the two sexes accompanied by the practice, is shown how the gender (relationship between sexes) is constitute in the differents and how is, to end of accounts, a cultural construction (Bourdieu, 1997; Lamas, 1992; Velásquez, 2003).

\section{Keywords}

Gender - education - field - habitus. 


\section{Introducción}

La intención de este trabajo ${ }^{1}$ es reflexionar sobre la desigualdad de género en el espacio escolar, particularizando la mirada a partir de la indagación de campo en la escuela rural de la localidad de Berisso.

Cabe comenzar por una explicitación del posicionamiento teórico: la visión sobre la noción de género con la que se comenzó la indagación se correspondía con aquella que observa el problema desde una mirada sustancialista, que atraviesa el sentido común, lo aparente, lo dado de las cosas de una vez y para siempre. La profundización en el tema y en la bibliografía produjeron la necesidad de interpelar las miradas tan arraigadas y naturalizadas en el mundo social sobre el hombre y la mujer, la forma de ser del alumno y alumna, y en este sentido me pregunto: ¿existe una forma y mejor aun existe una única forma?, es decir, ¿cuál es el imaginario presente acerca de ello?, ¿cuál es la actitud que se espera de un niño o una niña?

En oposición a esa mirada sustancialista, se adhiere a los posicionamientos teóricos de Lamas (1992) y Velásquez (2003), quienes aludiendo a la relación entre los sexos expresan que el género es una construcción cultural de la diferencia sexual. Por lo tanto, puede establecerse que este, como todo concepto relacional, implica la relación entre el mundo de las mujeres y hombres, que son constitutivos de cualquier sistema social interactuando aspectos psicológicos, culturales, sociales de la masculinidad y feminidad.

Sobre la base central de las categorías de campo y habitus planteadas por Bourdieu e indagando acerca de las características que toma el género en el campo educativo se lleva a cabo una reflexión acerca de la manera en que la escuela es generadora de habitus.

Este enfoque relacional permite mostrar que al hablar del mundo de la mujer no se puede desconocer el mundo del hombre, así como la masculinidad de la feminidad, dado que son procesos que se dan en un entramado complejo que constituye una totalidad social e histórica.

\section{Desarrollo conceptual}

Bourdieu (2008) "...las piernas, los brazos están llenos de imperativos adormecidos. Y uno no terminaría nunca de enumerar los valores hecho cuerpo por la transustanciación operada por la clandestina persuasión de una pedagogía implícita, capaz de inculcar toda una cosmología, una ética, una política a través de mandatos

\footnotetext{
${ }^{1}$ Versión revisada y mejorada del trabajo "Algunas categorías de Bourdieu para pensar las relaciones de género en el espacio educativo". Presentado en seminario: la perspectiva de Bourdieu: herramientas para el análisis del mundo social. Facultad de Trabajo Social- Universidad Nacional de La Plata. 2011.
} 
tan insignificantes como 'estate derecho' (...) y de inscribir en los detalles en apariencia más insignificantes del vestir, de la compostura o de las maneras corporales y verbales los principios fundamentales de la arbitrariedad cultural, situados así fuera de la conciencia y explicitación...", p. 112.

\section{La mujer en el campo educativo}

El acceso de las mujeres al sistema educativo no fue siempre posible. Tal como expresa Silva (2001), son ellas — sobre todo en los países periféricos del capitalismolas que tenían un nivel menor de instrucción con respecto a los hombres. A ello se sumaba, además de los condicionamientos sociales, que luego de haber ingresado en el sistema educativo se restringían las posibilidades de elección de ciertas carreras universitarias que estaban vedadas para mujeres, tal es el caso de la medicina.

La imposibilidad de las mujeres de ingresar en la educación no fue solo una realidad que caracterizó a Argentina. Cortazzo (1988) señala que es recién en 1879 cuando se abren las instituciones de enseñanza superior a las mujeres. Dice la autora citada que

"en Brasil, en 1887, Rita Lobato Velho Lopes es la primera mujer que recibe el título de médica (...) en el mismo período se reciben abogadas pero no les es permitido ejercer (...). En 1889, [en Argentina] se gradúa la primera mujer médica, Cecilia Grierson, pero sólo le fue permitido ejercer tres años más tarde. Pocos años después María Angélica Barrera obtuvo el grado de abogada..." (Traducción mía).

El contexto en el que se dio el ingreso de las mujeres en el sistema educativo, a fines del siglo XIX, estuvo basado en mitos y prejuicios. A las mujeres se les negaban sus capacidades intelectuales y se las ubicaba en el ámbito doméstico y de procreación desde un lugar de inferioridad reducido al cuidado de los niños y a los quehaceres del hogar, "ayudando" al hombre, quien realizaba el trabajo productivo y era el responsable de satisfacer monetariamente a la familia. ${ }^{2}$ Según lo desarrollado por Nari (1995); Cortazzo (2001) y Cortazzo, Schettini $(2002,2004)$, el ámbito por excelencia de la mujer era el privado, y ella poseía ciertas actitudes (paciencia, pasividad, dulzura) que venían a complementar al hombre fuerte, valiente e inteligente. Esta división de espacios (público-privado/intelectual-moral) y complementariedad justificaba y legitimaba la subordinación de la mujer respecto del hombre.

De ahí que las virtudes "propias" de las funciones y usos del cuerpo femenino y masculino se vinculan, considerando a Bourdieu (2008), con la división del trabajo entre los sexos y al mismo tiempo con la división sexual de trabajo. En este sentido se explican

\footnotetext{
${ }^{2}$ Ello se construye como los roles tradicionales de la mujer y del hombre respectivamente.
} 
la división de tareas y funciones asignadas cultural y socialmente a la mujer y al hombre y complementariamente los espacios habilitados desde el nacimiento y los primeros años de vida que incorporan y objetivan los esquemas clasificatorios que inscriben y definen socialmente la división sexual del trabajo y la división del trabajo entre sexos. ${ }^{3}$

En los primeros años de conformación del sistema educativo, el Estado, a través de sus aparatos ideológicos (como la educación) pretendía no solo la incorporación de las masas al modelo productivo, sino también la apropiación de valores y principios que hicieran viable la integración de los grupos de inmigrantes para lograr una identidad nacional. Pensar la incorporación de la mujer en la educación permite analizar, a partir de la noción de campo ${ }^{4}$ trabajada por Bourdieu (1990), las relaciones de fuerza que se circunscriben alrededor del género y la educación, entendiendo que hay algo por lo que se lucha, un acuerdo (illusio) que indica que vale la pena disputarlo, se pone en juego el capital económico, cultural y simbólico, con intereses específicos de cada grupo y agentes dispuestos a jugar.

$\mathrm{Si}$, tal como establece Bourdieu, los agentes que juegan en el campo por una distribución y acumulación de capital en los espacios del mundo social y aquellos que intentan mantener las mismas estructuras están dotados de un habitus, ${ }^{5}$ entonces tener un conocimiento y reconocimiento de quiénes están y qué está en juego permite conocer las percepciones y forma de ver y actuar en y sobre la realidad social. El campo refleja la lucha de fuerzas: están quienes intentan mantener el statu quo y aquellos que promueven la transformación de la estructura de distribución de capital en el campo (en términos de Bourdieu ortodoxia y herejía, respectivamente). Para ello diseñan estrategias de conservación y subversión respectivamente. Dentro de las estrategias de resistencia que adoptaron las mujeres para modificar las posiciones tradicionalmente asignadas, se vincularon con la conquista de nuevos espacios mediante luchas y la organización de movimientos feministas.

Estos procesos societales fueron construyendo cierta idea de masculinidad y feminidad, desde el modelo binario de hombre-mujer heterosexual (según la anatomía biológica) se configuraron determinadas formas de habitar y ser del cuerpo femenino y masculino, en las cuales los/las que no siguen estos parámetros social y culturalmente aceptados son considerados desviados, anormales, etc. En este sentido, Bourdieu (1997) plantea:

\footnotetext{
${ }^{3}$ Las instituciones por excelencia de socialización y objetivación: la familia y la escuela.

${ }^{4}$ Bourdieu (1987) define el campo social como "Espacios de juego históricamente constituidos con sus instituciones específicas y sus leyes de funcionamiento propias", p. 108.

${ }^{5}$ Según lo expresa Bourdieu (2008) "... El habitus es un sistema de disposiciones duraderas y transferibles, estructuras estructuradas predispuestas a funcionar como estructuras estructurantes, es decir, como principios generadores y organizadores de prácticas y de representaciones que pueden ser objetivamente adaptadas a su meta sin suponer el propósito consciente de ciertos fines ni el dominio expreso de las operaciones necesarias para alcanzarlos, objetivamente 'reguladas' y 'regulares' sin ser para nada el producto de la obediencia a determinadas reglas y, por todo ello, colectivamente orquestadas sin ser el producto de la acción organizadora de un director de orquesta", p.86.
} 
"El modo de pensamiento sustancialista que es el del sentido común y que conduce a tratar las actividades o las preferencias propias de determinados individuos o determinados grupos de una sociedad determinada en un momento determinado como propiedades sustanciales inscriptas de una vez y para siempre en una especie de esencia biológica o cultural..." p. 11.

En este sentido, Conway (1998) expresa que los sistemas de género ${ }^{6}$ a lo largo de la historia han sido binarios, lo que ha cambiado es el significado que se les ha dado. Estas antinomias remiten a procesos culturales y sociales más amplios, en los que las diferencias entre las mujeres y hombres no son visibles ni claras, y es en ello donde reside su poder, eficacia y relevancia.

\section{Algunas consideraciones del género en el campo educativo contemporáneo. Estudio de un caso particular para pensar la generalidad}

En la actualidad, en el campo educativo, respecto de los contenidos curriculares, no está tan pronunciada la diferenciación de materias para mujeres (referidas aprender a bordar, coser) y varones (pintura, carpintería, electricidad); lo que sí se observa son actividades distintas dentro del espacio de Educación Física, vinculando estas diferencias con la fuerza y con los deportes; qué "pueden hacer" y qué "son" actividades propias de las mujeres o cuáles lo son de los varones. Aquí se evidencian los prejuicios y estereotipos de lo que es ser mujer y varón estableciéndose lo que es posible y lo que está prohibido para cada uno.

Por otra parte, de acuerdo con los patrones comunes de emoción en la institución educativa, la mujer está habilitada a llorar, ponerse triste, pero el varón con esas mismas actitudes es considerado por sus mismos compañeros como "mariquita". Por otra parte, este espacio sigue reproduciendo la heterosexualidad como única opción. Un ejemplo de ello es cómo se organizan los actos educativos y sus contenidos en las principales fechas patrias. Estos actos patrios, como el Día de la Independencia, el Día del Maestro, etc., refuerzan este modelo binario con un destacado protagonismo del hombre héroe y la invisibilización de la mujer.

Estas situaciones que nos remiten a la noción de género son analizadas a la luz de los procesos singulares de la institución educativa de Berisso. ${ }^{7}$ Los estudiantes no

\footnotetext{
${ }^{6}$ Conway (1998) define que "Los sistemas de género son sistemas binarios que oponen la hembra al macho, lo masculino a lo femenino, rara vez sobre la base de la igualdad, sino, por lo general, en términos jerárquicos...", p. 117.

${ }^{7}$ La escuela rural La Hermosura pertenece al municipio de Berisso y depende de la Dirección General de Educación. A lo largo de su historia institucional tuvo cambios de denominación y ubicación, y desde 1957 la escuela mantiene la misma denominación. Considerando el marco legal, por la distancia a la que se encuentra la escuela se la caracteriza como rural, pero debido a la cantidad de chicos que asisten y sus particularidades, no es pertinente esta denominación. En los
} 
provienen de zonas rurales, ya que asisten a la escuela chicos de la localidad de La Plata y de Berisso, barrio La Franja. Son en su mayoría de nacionalidad paraguaya, boliviana y peruana. Los padres o de quienes están a cargo son mayoritariamente albañiles o vendedores ambulantes, y en su mayoría las madres, amas de casa o trabajadoras domésticas, quienes no alcanzan a cubrir las necesidades básicas. ${ }^{8}$ Es así que ellos son destinatarios de políticas sociales, como la Asignación Universal por Hijo para la Protección Social y Servicio de Alimentación Escolar; dichas políticas diseñadas con el objetivo de mejorar la situación de vulnerabilidad económica y social en la que están sumergidos estos agentes (Agis y otros 2010).

Dadas las características de las niñas y niños que concurren a la institución, a decir de Domenech (2004), se establece una relación entre la población que concurre, el lugar donde se ubica la institución y por otra parte la infraestructura y los servicios que esta institución rural presenta. Esta caracterización de la población nos permite pensar la dominación respecto de la posición de inferioridad de la mujer en relación con el tipo de tarea y funciones a la que está directamente vinculada, pero también cómo se acrecienta la desigualdad considerando la posición de subordinación que ocupa en el espacio social, es decir, si agregamos también la clase social a la que pertenece.

Por lo tanto, la producción de formas culturales - conductas femeninas y masculinas - está mediada por una serie de instituciones, entre ellas la educativa, que interactúan complejamente; es por ello que el género no puede desvincularse de la clase social, raza, cultura. En muchos casos la inclusión de algunos de estos aspectos llega a acrecentar la desigualdad. En este sentido se destaca la importancia de describir la posición de la escuela en el espacio social y al mismo tiempo describir las condiciones materiales de los agentes que concurren a la institución como elementos que favorecen la construcción de cierta masculinidad y feminidad desde este escenario.

Resumiendo: "Las determinaciones sociales ligadas a una posición determinada en el espacio social tienden a modelar a través de la relación con el propio cuerpo, las disposiciones constitutivas de identidad sexual $y$, sin duda también, las disposiciones sexuales mismas" (Bourdieu, 2008: 115). Este fragmento señala cómo las disposiciones de los sexos y la conformación de la identidad sexual está determinada socialmente, teniendo como horizonte la posición que se ocupa en el espacio social.

\footnotetext{
comienzos de la escuela, el edificio dependía del propietario de la tierra del campo, el Estado pagaba un monto estipulado por mes destinado al salario del director. En 1924, este edificio escolar pasó a depender del Consejo Escolar. Esta situación significó una mayor intervención o preocupación estatal sobre el área educativa. Información extraída del Libro de oro (2008)..Es un libro artesanal elaborado por alumnos, docentes y exalumnos, reconstrucción de "producto y productores de la historia de la escuela".

${ }^{8}$ Necesidades básicas insatisfechas es un concepto descriptivo para medir la pobreza, específicamente la situación de los pobres estructurales. Expresa las carencias materiales de determinados servicios considerados básicos. Frente a la crítica de ese concepto, Gutiérrez (2004) propone pensar la pobreza en términos relacionales de bienes de "lo que se tiene" y no únicamente de necesidades, de "lo que se carece".
} 
El contexto de los años 90 en el que se desarrolla la educación y otras áreas de la vida social refuerza el modelo económico basado en el libre mercado. Los organismos internacionales adquieren un mayor protagonismo sobre la educación de la población, desresponsabilizando al Estado como principal garante de los derechos indispensables para el crecimiento y desarrollo de los sujetos. Las características de este período Estado Neoliberal- son la descentralización, la focalización y la inversión en actividades consideradas productivas, en las cuales los chicos pobres de escuelas pobres van a ser los que reciban menor cantidad y calidad de recursos.

Hoy la escuela cuenta con una matrícula de 124 educandos en un espacio muy reducido. Localizada sobre el camino a Magdalena, se presentan dificultades para gozar de algunos servicios básicos. Las deficiencias -escasez de transporte público, imposibilidad de acceso al teléfono público, el espacio reducido para la práctica pedagógica- ${ }^{9}$ que se presentan no intentan ser un mero dato descriptivo-anecdótico, sino, por el contrario, reflejan las condiciones en las que se encuentra esta institución educativa (que no son ajenas a las realidades que viven otras escuelas) y, por otra parte, caracterizan a los agentes que constituyen la institución. Estos déficits solo pueden ser resueltos o mejorados con la participación de distintos agentes institucionales, y por lo tanto con la decisión política para poder concretarlo. A decir de Gutiérrez (2007), la cantidad y calidad de servicios y recursos que conforman a este espacio educativo expresan que "el universo de los posibles no es igualmente posibles para todos" p. 21. En este sentido, este fragmento hace referencia no solo a la distancia geográfica entre la existencia de distintos servicios, sino también la distancia social real que posibilita crear las condiciones objetivas que garanticen el acceso a los servicios básicos.

Desde lo trabajado por Bourdieu (2007), las condiciones objetivas que posibilitan acceder a los servicios para garantizar el bienestar de los aprendices y sus familias permiten analizar las relaciones que existen en el espacio social, particularmente el educativo, entre las condiciones de reproducción de las situaciones de pobreza en las

\footnotetext{
${ }^{9}$ En cuanto a los servicios de comunicación que presenta la zona en la que se ubica la institución, hasta hace un año fue escasa la presencia de transporte público, es decir, además de que pasaba por la escuela una sola línea de colectivo, su presencia era insuficiente ocasionando que en algunas circunstancias los educandos deban quedarse en la institución donde no cuentan con un lugar apropiado. Ante esta situación se conformó una comisión barrial con el objetivo de que ingresen más micros. A más de un año de reclamo, se incorporaron dos recorridos continuando la misma línea de colectivo. Son relevantes estas acciones colectivas que tienen que construir los agentes frente a la deficiencia del servicio de transporte público, porque en la mayoría de los casos es el único medio que permite conectar a los sujetos con otros espacios de la ciudad. Por otra parte, otro de los reclamos de la institución es el teléfono público, ya que la línea de teléfono que hay para comunicarse es mediante un teléfono celular que en muchas ocasiones no tiene señal y en caso de urgencia los distintos actores institucionales quedan incomunicados.

Otra de las deficiencias que presenta la institución es el espacio reducido para el funcionamiento de la escuela. A pesar de que se realizan actividades de distinta índole, no hay que olvidar que la escuela se construyó con una determinada capacidad y hoy excede el número correspondiente. Entonces la matrícula de la institución aumenta, pero la infraestructura no acompaña ese desarrollo. Esta descripción es importante considerando que el espacio edilicio es uno de los factores indispensables —además de los recursos materiales, humanos_- para desarrollar las actividades que competen al proceso de enseñanza-aprendizaje.
} 
que están sumergidos estos agentes que concurren a la escuela, pero al mismo tiempo los vínculos y estrategias que construyen con otros grupos (del poder político) para mejorar mínimamente las condiciones de existencia. Es allí donde la política social cumple un lugar muy importante, que en la escuela se traduce en el aumento de los recursos destinados para enseñar y aprender dentro y fuera de la institución, y por otra parte, en forma complementaria los recursos destinados básicamente para la subsistencia (como la alimentación que reciben los niños a través del comedor).

El diseño e implementación de distintas actividades (visitas guiadas y viajes educativos, teatro, elaboración de productos artesanales) que trascienden el espacio áulico refleja la forma en que se desarrolla el proceso enseñanza-aprendizaje. Según describe Pogré (2007):

"El aprendizaje es un proceso complejo en el que cada sujeto resignifica la realidad a partir de una reconstrucción propia y singular. Esto significa que el aprendizaje no es algo que se 'tiene o no se tiene', como una posesión acabada. Es un proceso y, además, cada sujeto realiza este proceso de un modo propio y singular. Con esto queda rota la fantasía de la homogeneidad y del pensar la clase para el 'alumno medio' o 'el común de los estudiantes', como si hubiese un modo 'patrón' de aprender" p. 28.

Este fragmento textual hace hincapié en que no hay una sola forma de aprender, dado que cada cual aprehende y aprende de manera singular, ya sea los contenidos curriculares como aquellas actividades con las que también se pretende enseñar. Ese aprendizaje está condicionado por la trayectoria social, cultural, política y económica del contexto, que expresa y constituye ese sujeto singular.

Algunos rasgos que atraviesan a la institución están dados por las costumbres, cultura y el lenguaje. En este caso la mayoría de los niños/as habla guaraní, lo que en algunas situaciones se presenta como un obstáculo en el proceso de enseñanza aprendizaje. Las maestras remiten a la dificultad de los niños para expresarse en el aula o que directamente no hablan. Esto último ¿podría reflejar que en el interior de la escuela circule la lengua "socialmente aceptada", distinta de la propia?; ¿cómo es la comunicación entre los padres de los niños y los directivos, docentes?, dado que las dificultades de comunicación por habla diferente no son solo de los niños, sino también de los padres.

La dificultad en la comunicación con distintas lenguas no es solo una situación del niño que asiste a la escuela, sino que es en el contexto familiar donde se fomenta la invisibilización del habla originaria. Este contexto familiar está atravesado por procesos sociales, políticos, culturales, económicos más generales que condicionan la forma de relacionarse y actuar. 
La necesidad de poder contextualizar a ese niño que llega y permanece en la institución radica en que muchas veces los enunciados de los programas escolares construyen lo "cierto" de una cultura como "verdad" y "único", sin tomar en cuenta las particularidades de los grupos poblacionales, generando estigmatización, discriminación sobre aquellos y aquello que "no encuadra" dentro de los parámetros establecidos.

Es así que los contenidos curriculares que se configuran desde la educación formal no incorporan los saberes de los educandos y sus familias, alfabetizados en el guaraní. Pueden hablar, escribir y leer la lengua guaraní, no así la lengua oficial. Es importante resaltar la mirada de los padres sobre sus hijos para que se alfabeticen en español "... poder ser alguien, seguir estudiando y no repetir la misma vida que nosotros" (fragmento de la madre de un estudiante $)^{10}$. Esa expresión se vincula con el concepto que trabaja Bourdieu de capital cultural y simbólico ${ }^{11}$, y evidencia el reconocimiento y la legitimidad que tiene en sus vidas el tránsito por el sistema educativo que permite dar "existencia" a un agente. Además esa expresión se centra en una idea de progreso o ascenso social desde una realización personal, individual, sin tomar en consideración la cultura, el contexto socio-político y económico que hacen posible continuar en el sistema educativo o la inserción en el mercado de trabajo.

En este marco, la forma de crear la masculinidad —que se origina en procesos societales y culturales más generales y se particulariza, por ejemplo, en la Escuela de Berisso-, expresa Scharagrodsky (2007), está atravesada por su oposición a la feminidad, por ser heterosexual, y su reticencia y negación a la homofobia. Las situaciones de rechazo a la homofobia se evidencian en los distintos espacios de la escuela cuando los mismos directivos estipulan una vestimenta diferenciada para los varones y las mujeres o cuando entre los mismos varones "la pelea" toma el símbolo de fuerza o se lo designa valiente; y esta misma situación es impensada para el grupo de las mujeres. Ello permite analizar la noción de habitus, principio generador y unificador de una posición que caracteriza un estilo de vida de un grupo de personas, bienes y prácticas. Bourdieu (1988) manifiesta que el habitus funciona como un "... esquema de producción de prácticas y un sistema de esquemas de percepción y apreciación de prácticas..." p. 134. Esto implica que el habitus configura un mundo social que parece

\footnotetext{
${ }^{10}$ Extraído de una reunión entre la directora, el maestro y la madre de un alumno, para buscar alguna solución frente a la desatención y dispersión de este último en el aula. Particularmente frase emitida por la madre.

${ }^{11}$ El capital cultural según Bourdieu (2006) puede existir en tres formas o estados: 1) interiorizado o incorporado, es decir, en forma de disposiciones duraderas del organismo; 2) en estado objetivado, en forma de bienes culturales, cuadros, libros que son resultado y muestra de disputas intelectuales y, por último, el tercer estado, institucionalizado en forma objetivada a través de títulos académicos. Este concepto lo utilizó el autor para explicar la desigualdad del rendimiento escolar de niños de diferentes clases sociales. El mismo autor (2008) expone que el capital simbólico es la "diferencia reconocida, aceptada como legítima y que procura un signo de distinción, manifiesto en los estilos de vida", p. 26.
} 
evidente, dado, ya construido, asociado a prácticas y representaciones en determinada posición social disponibles para clasificar.

Lamas (1996) menciona que "... El sujeto social es producido por las representaciones simbólicas. El hombre y la mujer no son reflejo de una realidad natural, sino el resultado de una producción histórica y social", p. 343. Ello expresa que las masculinidades y feminidades que se manifiestan en la cotidianidad no son fenómenos inmutables, que siempre se expusieron de una manera unívoca en el ámbito escolar; de lo contrario son producto y productores de múltiples prácticas, representaciones, símbolos, códigos construidos por hombres y mujeres a lo largo de la historia.

Por lo tanto, la escuela es producto de y también produce determinados sentidos, representaciones acerca de lo que son la mujer y el varón, de lo que es ser femenino y masculino, qué vestimenta, pose, conducta, lenguaje tiene que tener una mujer y un varón. Esto forma parte de lo que Nicastro (1997) llama cultura organizacional, es necesario conocer los códigos formales e informales para luego de haber ingresado poder ser admitido y permanecer en la institución.

Una dimensión importante que se pone en juego en la cuestión de género en el campo simbólico es la lucha simbólica por la forma de percibir el mundo social. A decir de Bourdieu (1990), esta percepción de la realidad social es resultado de una doble estructuración: una objetiva y otra subjetiva. Estas dos dimensiones están interconectadas entre las propiedades de los agentes e instituciones que se vinculan con las percepciones; estas últimas y las apreciaciones de los agentes son producto de luchas simbólicas históricas. Esta lucha simbólica a la que hace referencia Bourdieu (1990) pone de manifiesto la lucha por el monopolio de la nominación legítima como imposición oficial, "acto de imposición simbólica que cuenta con toda la fuerza de lo colectivo, del consenso, del sentido común, porque es operada por un mandatario del Estado, detentador del monopolio de la violencia simbólica legítima" p. 294.

Las prácticas, representaciones sociales que circulan en los pasillos de la escuela y son naturalizadas, posibilitan el ejercicio de la violencia simbólica definida por Bourdieu en el escrito de Lamas (1996) como "... aquella violencia que se ejerce sobre un agente social con su complicidad o consentimiento". La violencia simbólica es un tipo de violencia que está más invisibilizada, y en ello opera su eficacia. Se materializa en las relaciones que se construyen día a día, cuando se imponen ciertos contenidos o cuando se clasifican las actividades según el sexo.

Por lo tanto, las relaciones de poder también se ejercen, por ejemplo, entre los maestros cuando hay algunos que tienen más años en la institución ("los más antiguos son los que más saben", imaginario que circula en la escuela, y esto es aceptado así) y el mayor conocimiento de los códigos de la institución los ubica en un lugar de poder. A su vez, estas relaciones se hacen muy notables entre los maestros y los alumnos desde el contenido que se asigna a cada clase (qué se da y qué no) hasta el lugar de 
autoridad que posee y ejerce el maestro en la institución- y entre los mismos alumnos. Esto me remite a lo que Bourdieu (1990) denomina punto de vista autorizado de un agente autorizado, es decir, el punto de vista de un actor institucional que es reconocido y legitimado, autoridad representante del Estado.

Chaui (1989) manifiesta que "el discurso competente es aquel que puede ser dicho, oído o aceptado como verdadero o autorizado (estos términos ahora se equivalen) porque perdió los lazos con el lugar y el tiempo de su origen... El discurso competente es el discurso instituido... El discurso competente se confunde, entonces, con el lenguaje institucionalmente permitido o autorizado, es decir, con el discurso en el cual los interlocutores ya fueron previamente reconocidos como teniendo el derecho de hablar y oír...". p. 7. (Traducción mía).

Las instancias en las que se seleccionan los juegos que son para las mujeres y los que son para los hombres, como también la elección diferencial de la vestimenta, permiten pensar cómo el género es un área a través de la cual circula el poder, en este caso con un carácter fuertemente simbólico. Coincidiendo con Bourdieu en Gutiérrez (2000):

"... El poder es constitutivo de la sociedad y, ontológicamente, existe en las cosas y en los cuerpos, en los campos y en los habitus, en las instituciones y en los cerebros (como diría Marx). Por lo tanto el poder existe físicamente, objetivamente, pero también simbólicamente". p. 10.

\section{Reflexiones finales}

Según lo describe Bourdieu (1990)

"... las categorías de la percepción del mundo social son, en lo esencial, el producto de la incorporación de las estructuras objetivas del espacio social. En consecuencia, inclinan a los agentes a tomar el mundo social tal cual es, a aceptarlo como natural más que a revelarse contra él, a oponerle mundos posibles, diferentes, y aun antagonistas...". p. 289.

En este sentido, se destaca la importancia de desnaturalizar e interpelar las prácticas que se tejen cotidianamente sobre lo que se espera social y culturalmente que la mujer y hombre piensen, sientan, actúen para comenzar a pensar nuevas y múltiples formas de habitar los cuerpos. Por eso es indispensable historizar y desencializar los modelos patriarcales que se han instalado con tanta fuerza en la sociedad, y que organizan los distintos ámbitos de la vida cotidiana. Pensar en ello implica comenzar a desnaturalizar la heterosexualidad como única opción posible, desmitificar que hay una forma de ser mujer y hombre, para poder trascender con el binarismo y construir nuevos sentidos a lo que se configura como diferencia sexual. Es por ello que la sociedad existe en y por 
el conflicto, conflicto entre visiones y percepciones del mundo simbólico que intentan imponer divisiones legítimas.

En este recorrido es necesario destacar el concepto que expone Cavana (1995) de diferencia no en términos negativos, es decir, de inferioridad, dominación, subordinación, sino en el sentido de reconocer las particularidades de cada grupo social en forma complementaria y responder a las necesidades de las mujeres y de los hombres. Es un desafío trabajar sobre esta temática, dado que exige revisar las propias percepciones, prácticas y representaciones que tan internalizadas se encuentran (ello se debe a su eficacia simbólica) para no seguir reproduciéndolas y reforzar mecanismos discriminatorios, y en pos de ello configurar nuevos sentidos que incluyan desde un lugar más equitativo a las mujeres y a los hombres. Esto implicará interpelar nuestras acciones cotidianas, y para ello Bourdieu (2008) y Gutiérrez (2000) sugieren dos caminos para recorrer: la reflexividad y la objetivación del sujeto objetivante como herramientas para poner atención y en tensión la relación que se construye entre el investigador y aquellos agentes, situaciones con las que se investiga, y desarrollar una vigilancia constante con el proceso mismo de investigación. 


\section{Bibliografía}

Agis, E., Cañete, C. y Panigo, D. (2010). El impacto de la Asignación Universal por Hijo en Argentina. Buenos Aires: Mimeo.

Bourdieu, P. (1988). El interés del sociólogo. En: Cosas dichas (pp. 108-114). Buenos Aires: Gedisa.

-(1988). Espacio social y poder simbólico. En: Cosas Dichas (pp. 127-142).

Buenos Aires: Gedisa.

-(1990a). Algunas propiedades de los campos. En: Sociología y cultura (pp. 135-141). México: Grijalbo.

-(1990b). Espacio social y génesis de las clases. En: Sociología y Cultura (pp. 281-310). México: Grijalbo.

-(1997a). Espacio social y espacio simbólico. En: Razones prácticas, sobre la teoría de la acción (pp. 11 - 32). Barcelona: Anagrama.

(2006). Los tres estados del capital cultural. En: Campo del poder y reproducción social. Elementos para un análisis de la dinámica de las clases (pp. 195202). Córdoba: Ferreyra Editor.

--(2008). El sentido práctico (Cap. III y IV). Buenos Aires: Siglo XXI

Cavana, M. (1995). Diferencia. En: C. Amoros (comp.). Diez palabras claves sobre mujer (pp. 85-118). Navarra: Laqueaur.

Chaui, M. (1989). Cultura y democracia. São Paulo Brasil: Editora Cortez.

Conway, J. (1998). El concepto de género. En: Marysa Navarro Y Catharine Stimpson (Comp.) ¿Qué son los estudios de mujeres? (pp. 167-178). Buenos Aires: FCE.

Cortazzo, I. (1988). Mujer y trabajo. Forma parte del informe de Investigación Mulheres trabalhadoras da indústria textil e saúde: investigação para a ação no Rio Grande do Sul e Santa Catarina (Brasil) e na Capital Federal e Provincia de Buenos Aires (Argentina). Porto Alegre Brasil: CNPq Conselho Nacional de Desenvolvimento Científico e Tecnológico Brasil (Inédito).

Cortazzo, I. (2001). O trabalho da mulher. Uma desigualde encoberta?. En: Mulher e Trabalho (Parte II pp. 55-61). Publicação Especial do convenio da pesquisa de emprego e desemprego na Região Metropolitana de Porto Alegre- PED-RMPA/ Irene M.S. Galeazzi organizadora. Porto Alegre: FEE Fundação de Economía e Estatística Siegrfried Emanuel Heuser; Fundação Sistema Estadual de Análise de Dados SP; DIESSE; Fundação Gaúcha do Trabalho e Ação Social; Sistema Nacional de Emprego. INSS 1519-8820.

Cortazzo, I; Schettini, P. (2002). Uma pesquisa, na Argentina, sobre um velho fenómeno: a inclusão da mulher no trabalho. En: Mulher e Trabalho (pp. 119-129). Publicação Especial do convenio da pesquisa de emprego e desemprego na Região Metropolitana de Porto Alegre- PED-RMPA/ Irene M.S. Galeazzi organizadora. Porto Alegre: FEE Fundação de Economía e Estatística Siegrfried Emanuel Heuser; Fundação 
Sistema Estadual de Análise de Dados SP; DIESSE; Fundação Gaúcha do Trabalho e Ação Social; Sistema Nacional de Emprego. INSS 1519-8820.

--------(2004). Mujeres Pobres en la trampa Cultural de la Política Social y Local. En: Strey, M; Lisboa Cabeda, Prenh, D Estudos de: Género: e Questões Contemporâneas (pp.73/101). EDIPUCRS. Brasil: Pontificia Universidade Católica de Rio Grande do Sul. ISBN 85-7430-442-5.

Domenech, E. (2007). El banco mundial en el país de la desigualdad: políticas y discursos neoliberales sobre diversidad cultural y educación en América Latina. En: A. Grimson (comp.) cultura y neoliberalismo (pp. 61-89). Buenos Aires: Consejo Latinoamericano de Ciencias Sociales (CLACSO).

Foucault, M. (1992). Microfísica del poder (Puntos 6, 8, 9 y 10). Madrid: La Piqueta.

Gutiérrez, A. (2004b). Estrategias de reproducción social en la pobreza. En: Pobre como siempre (pp. 21-62). Córdoba: Ferreyra Editor. (2007). Herramientas teórico-metodológicas de un análisis relacional para los estudios de la pobreza. Revista Ciencia, Docencia y Tecnología, núm. 35, 15-33. Paraná: uner.

(2008). Clases, espacio social y estrategias: una introducción al análisis de la reproducción social. En: Bourdieu, P. (2007). Campo del poder y reproducción social. Elementos para un análisis de las clases sociales. Córdoba: Ferreyra Editor.

Lamas, M. (1996). Usos, dificultades y posibilidades de la categoría "género". En: Lamas (Comp.) El género: la construcción cultural de la diferencia sexual (pp. 327-366). México: Porrúa.

Nari, M. (1995). La educación de la mujer. Revista Mora, núm. 1, 31-45. Buenos Aires.

Nicastro, S. (1997). Su registro en la cultura e influencia en el funcionamiento de la institución educativa. En: La historia institucional y el director en la escuela (pp. 61- 86).

Buenos Aires: Paidós.

Pogré, P. (2007). "¿Cómo enseñar para que los alumnos aprendan?". Revista Diálogo Educacional, vol. 7, núm. 20, pp. 25-32. Brasil: Curitiba.

Silva, T. (2001). Las relaciones de género y la pedagogía feminista. En: Espacios de Identidad. Una introducción a las teorías del currículum (pp. 111-119). Barcelona: Octaedro.

Scharagrodsky, P. (2007). Masculinidades valuadas y devaluadas. Tensiones, límites y posibilidades en el ámbito escolar, en: Baquero, R., Diker, G. y Frigerio, G. (Comp.) Las Formas de lo escolar (pp. 263-284). Buenos Aires: Del Estante Editorial.

Velásquez, S. (2003). La violencia de género como violencias cotidianas. En: Violencias cotidianas, violencia de género. Escuchar, comprender, ayudar (pp. 23-33). Buenos Aires: Paidós. 